\title{
Disentangling the ISM phases of the dwarf galaxy NGC 4214 using [C II] SOFIA/GREAT observations *
}

\author{
K. Fahrion ${ }^{1}$, D. Cormier ${ }^{1}$, F. Bigiel ${ }^{1}$, S. Hony ${ }^{1}$, N. P. Abel ${ }^{2}$, P. Cigan ${ }^{3}$, T. Csengeri ${ }^{4}$, U. U. Graf ${ }^{5}$, V. Lebouteiller ${ }^{6}$, \\ S. C. Madden ${ }^{6}, \mathrm{R} . \mathrm{Wu}^{7, \star \star}$, and L. Young ${ }^{8}$ \\ 1 Institut für Theoretische Astrophysik, Zentrum für Astronomie der Universität Heidelberg, Albert-Überle-Straße 2, \\ 69120 Heidelberg, Germany \\ e-mail: fahrion@stud.uni-heidelberg.de \\ 2 University of Cincinnati, Clermont College, MCGP Department, Batavia, OH 45103, USA \\ 3 School of Physics and Astronomy, Cardiff University, Cardiff CF24 3AA, UK \\ 4 Max-Planck-Institut für Radioastronomie, Auf dem Hügel 69, 53121 Bonn, Germany \\ 5 I. Physikalisches Institut der Universität zu Köln, Zülpicher Straße 77, 50937 Köln, Germany \\ ${ }^{6}$ Laboratoire AIM, CEA Saclay, Orme des Merisiers, Bât. 709, 91191 Gif-sur-Yvette, France \\ 7 Department of Astronomy, The University of Tokyo, Bunkyo-ku, 113-0033 Tokyo, Japan \\ 8 Physics Department, New Mexico Tech, 801 Leroy Place, Socorro, NM 87801, USA
}

Received 19 July 2016 / Accepted 2 November 2016

\begin{abstract}
Context. The [C II] $158 \mu \mathrm{m}$ fine structure line is one of the dominant cooling lines in the interstellar medium (ISM) and is an important tracer of star formation. Recent velocity-resolved studies with Herschel/HIFI and SOFIA/GREAT showed that the [C II] line can constrain the properties of the ISM phases in star-forming regions. The [C II] line as a tracer of star formation is particularly important in low-metallicity environments where $\mathrm{CO}$ emission is weak because of the presence of large amounts of CO-dark gas.

Aims. The nearby irregular dwarf galaxy NGC 4214 offers an excellent opportunity to study an actively star-forming ISM at low metallicity. We analyzed the spectrally resolved [C II] line profiles in three distinct regions at different evolutionary stages of NGC 4214 with respect to ancillary $\mathrm{HI}$ and $\mathrm{CO}$ data in order to study the origin of the [C II] line.

Methods. We used SOFIA/GREAT [C II] $158 \mu$ m observations, H I data from THINGS, and $\mathrm{CO}(2 \rightarrow 1)$ data from HERACLES to decompose the spectrally resolved [C II] line profiles into components associated with neutral atomic and molecular gas. We use this decomposition to infer gas masses traced by [C II] under different ISM conditions.

Results. Averaged over all regions, we associate about $46 \%$ of the [C II] emission with the H I emission. However, we can assign only $\sim 9 \%$ of the total [C II] emission to the cold neutral medium (CNM). We found that about $79 \%$ of the total molecular hydrogen mass is not traced by CO emission.

Conclusions. On average, the fraction of CO-dark gas dominates the molecular gas mass budget. The fraction seems to depend on the evolutionary stage of the regions: it is highest in the region covering a super star cluster in NGC 4214, while it is lower in a more compact, more metal-rich region.
\end{abstract}

Key words. galaxies: dwarf - galaxies: star formation - galaxies: individual: NGC 4214 - ISM: lines and bands

\section{Introduction}

Star formation proceeds through the contraction and collapse of cold, dense molecular clouds. To study the first stages of star formation, observations of these molecular clouds and the knowledge of their conditions are of great interest. However, direct observations of the most abundant molecule in the cold molecular clouds, $\mathrm{H}_{2}$, is not possible because the $\mathrm{H}_{2}$ molecule has no dipole moment and can therefore not be observed in cold and dense environments. Instead, carbon monoxide is traditionally used to study the molecular phase of the interstellar medium (ISM) as CO forms under the conditions prevalent in cold molecular clouds. The lowest ground rotational transition $J=1 \rightarrow 0$ creates the most prominent line at $2.6 \mathrm{~mm}$

\footnotetext{
$\star$ Reduced SOFIA/GREAT data are only available at the CDS via anonymous ftp to cdsarc.u-strasbg. fr (130.79.128.5) or via http://cdsarc.u-strasbg.fr/viz-bin/qcat?J/A+A/599/A9

$\star \star$ International Research Fellow of the Japan Society for the Promotion of Science (JSPS)
}

(115.27 GHz), but various other transition lines can be observed as well. The reservoir of molecular hydrogen from which new stars form is usually estimated via the CO-to- $\mathrm{H}_{2}$ conversion factor $\alpha_{\mathrm{CO}}$ (or $X_{\mathrm{CO}}$ ). Nonetheless, the CO-to- $\mathrm{H}_{2}$ conversion factor is a controversial quantity as it seems to vary with metallicity (Bolatto et al. 2013; Poglitsch et al. 1995; Cormier et al. 2014; Narayanan et al. 2011; Glover \& Mac Low 2011). Furthermore, evidence for hidden molecular hydrogen ("CO-Dark Molecular Gas", Wolfire et al. 2010) has been found not only in the Milky Way (Langer et al. 2014; Planck Collaboration XIX 2011; Grenier et al. 2005), but also in other galaxies (Israel 1997; Madden et al. 1997; Requena-Torres et al. 2016).

These recent studies raise the importance of other tracers that might give insight into the conditions of the molecular content. One of the most promising tracers is the [C II] fine-structure line at $158 \mu \mathrm{m}$ emitted by singly ionized carbon. [C II] is usually the strongest far-infrared line, representing up to $1 \%$ of the continuum emission in the FIR regime (e.g., Stacey et al. 1991; Genzel \& Cesarsky 2000; Cormier et al. 2015). Being one 

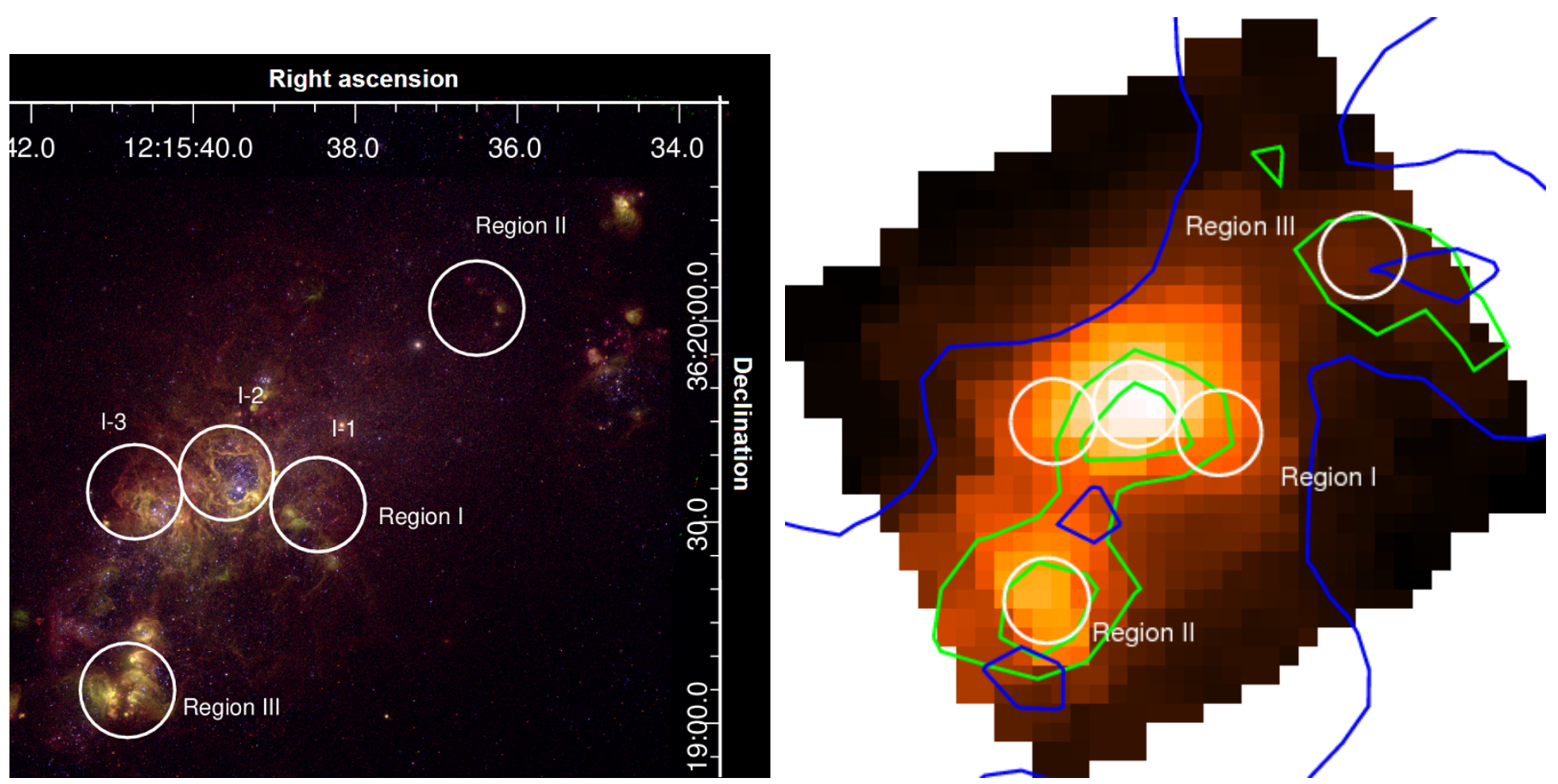

Fig. 1. Left: three-color image of the central part of NGC 4214 and pointing positions for SOFIA/GREAT observations. HST WFC3 filters $F 336 W$ (blue), F502 (green), and F657 (red) were used. Credit: NASA/Hubble. Right: integrated [C II] intensity map observed with Herschel/PACS (Cormier et al. 2010). H I (Walter et al. 2008) contours overlayed in blue, $\mathrm{CO}(2 \rightarrow 1)$ (Leroy et al. 2009) contours in green, and SOFIA/GREAT pointings as white circles with a diameter corresponding to $14.1^{\prime \prime}(\sim 200 \mathrm{pc})$. For $\mathrm{CO}(2 \rightarrow 1)$, the contour levels are at $1.2 \mathrm{~K} \mathrm{~km} \mathrm{~s}^{-1}$ and $2.4 \mathrm{~K} \mathrm{~km} \mathrm{~s}^{-1}$. For the extended $\mathrm{HI}$ the levels are at $0.23 \mathrm{Jy} \mathrm{beam}^{-1} \mathrm{~km} \mathrm{~s}^{-1}$ and $0.27 \mathrm{Jy} \mathrm{beam}^{-1} \mathrm{~km} \mathrm{~s}^{-1}$. The map covers a $1.6^{\prime} \times 1.6^{\prime}$ field of view $(\sim 1.3 \mathrm{kpc} \times 1.3 \mathrm{kpc})$.

of the dominant cooling lines in photon-dominated regions (PDRs, Hollenbach \& Tielens 1999) it is often used to study their conditions.

However, because ionized carbon dominates the carbon abundance under most conditions found in the ISM and the [C II] line can be excited by collisions with electrons, atomic, or molecular hydrogen, this line not only traces PDRs, but it can also be found in other phases of the ISM (e.g., Goldsmith et al. 2012). Therefore, the origin of the [C II] line has to be studied in detail in order to use it as a tracer of star-forming regions. Disentangling the different contributions from molecular, ionized, or atomic phases can be done by comparing the spectrally or spatially resolved [C II] emission with tracers of other ISM phases. As high spatial and spectral resolution is needed, this can only be done in nearby objects. So far, integrated line intensities have mostly been used to study star formation in external galaxies, but velocity-resolved observations of the Milky Way and nearby galaxies have become available with the HIFI instrument on Herschel and with the GREAT instrument on SOFIA. Constraining the amount of CO-dark molecular gas using [C II] is of particular interest for low-metallicity dwarf galaxies. These galaxies can have very weak CO emission (Schruba et al. 2012; Cormier et al. 2014). At the same time, dwarf galaxies can produce stars at rates that are normally found in starburst galaxies (e.g., Gallagher \& Hunter 1984; Hunter et al. 1989). These two observations combined imply that the CO-dark gas may outweigh the $\mathrm{CO}$-bright gas by a large factor. Measuring the $\mathrm{CO}$ dark gas fraction in low-metallicity dwarf galaxies is therefore of key importance for understanding their behavior.

In this paper, we take advantage of the new generation of velocity-resolved [C II] observations from SOFIA/GREAT to disentangle the origin of [C II] emission in NGC 4214.
NGC 4214 is a nearby irregular dwarf galaxy of Magellanic type at a distance of $2.9 \mathrm{Mpc}$ (Maíz-Apellániz et al. 2002; Dalcanton et al. 2009) with low metallicity $(\log (\mathrm{O} / \mathrm{H})+12=$ 8.2, Kobulnicky \& Skillman 1996). Owing to its proximity, there is a wealth of ancillary data for NGC 4214. CO(1 $\rightarrow 0)$ observations showed that NGC 4214 exhibits three distinct starforming regions in different evolutionary stages (Walter et al. 2001). The left panel in Fig. 1 shows a three-color image of the central part of NGC 4214 with the pointing positions of our SOFIA/GREAT observations of the star-forming regions overlayed as white circles. In the following, we use the labeling of the regions as shown in Fig. 1. The central region (region I) is the most evolved and is also the largest region, containing a super star cluster and several star clusters (Maíz-Apellániz 2001). Bright $\mathrm{O}$ stars as well as Wolf-Rayet stars and a $\mathrm{H} \alpha$ cavity are found in this region (MacKenty et al. 2000). Using a UVoptical survey of NGC 4214, Úbeda et al. (2007b) showed that region I is about $\sim 3-4 \mathrm{Myr}$ old. In contrast, the southeastern region, labeled as region II, is only $\sim 2 \mathrm{Myr}$ of age. This region is more compact and contains concentrated gas reservoirs (MacKenty et al. 2000) as well as OB stars and strong $\mathrm{H} \alpha$ emission, which is created in bright $\mathrm{H} \alpha$ knots (Úbeda et al. 2007a; Maíz-Apellániz et al. 1998). Using $\mathrm{H} \alpha$ images and H I velocity data obtained by McIntyre (1997), Thurow \& Wilcots (2005) found systematic differences in the kinematics of the ionized gas in region I and II. In the image, the southeastern region is labeled as region III. No star formation is recognizable in this region, but $\mathrm{CO}$ emission was found by Walter et al. (2001). Since this region is the least evolved, Walter et al. (2001) suggest that region III will form stars in the future. With these three distinct regions, NGC 4214 offers the possibility of studying the evolution of star formation at low metallicities and in different environments. 
Table 1. Fluxes of [C II], H I, and CO for the five SOFIA/GREAT pointing positions.

\begin{tabular}{ccccccccc}
\hline \hline Region & RA (J2000) & Dec (J2000) & $\begin{array}{c}F([\mathrm{C} \text { II }])_{\text {PACS }} \\
{\left[10^{-16} \mathrm{~W} \mathrm{~m}^{-2}\right]}\end{array}$ & $\begin{array}{c}F([\mathrm{C} \text { II }])_{\text {GREAT }} \\
{\left[10^{-16} \mathrm{~W} \mathrm{~m}^{-2}\right]}\end{array}$ & $\begin{array}{c}F(\mathrm{H} \mathrm{I}) \\
{\left[\mathrm{Jy} \mathrm{km} \mathrm{s}^{-1}\right]}\end{array}$ & $\begin{array}{c}F(\mathrm{CO}(2-1)) \\
{\left[\mathrm{Jy} \mathrm{km} \mathrm{s}^{-1}\right]}\end{array}$ & $\begin{array}{c}F(\mathrm{CO}(1-0)) \\
{\left[\mathrm{Jy} \mathrm{km} \mathrm{s}^{-1}\right]}\end{array}$ & $R_{\frac{2 \rightarrow 1}{\mathrm{I} \rightarrow 0}}$ \\
\hline I-1 & $12: 15: 38.48$ & $+36: 19: 32.4$ & $3.98 \pm 0.08$ & $3.54 \pm 0.49$ & $0.48 \pm 0.01$ & $10.27 \pm 2.21$ & $1.77 \pm 0.49$ & $1.46 \pm 0.52$ \\
I-2 & $12: 15: 39.61$ & $+36: 19: 37.1$ & $7.02 \pm 0.12$ & $9.62 \pm 0.43$ & $0.52 \pm 0.01$ & $16.64 \pm 2.08$ & $3.14 \pm 0.34$ & $1.33 \pm 0.22$ \\
I-3 & $12: 15: 40.74$ & $+36: 19: 34.3$ & $4.85 \pm 0.10$ & $7.27 \pm 0.40$ & $0.55 \pm 0.01$ & $6.76 \pm 1.95$ & $1.12 \pm 0.57$ & $1.52 \pm 0.89$ \\
II & $12: 15: 40.83$ & $+36: 19: 04.7$ & $5.03 \pm 0.09$ & $6.86 \pm 0.32$ & $0.49 \pm 0.01$ & $19.51 \pm 2.34$ & $6.76 \pm 0.39$ & $0.76 \pm 0.10$ \\
III & $12: 15: 36.52$ & $+36: 20: 01.7$ & $1.18 \pm 0.05$ & $2.49 \pm 0.62$ & $0.58 \pm 0.01$ & $12.09 \pm 2.73$ & $3.69 \pm 0.70$ & $0.88 \pm 0.25$ \\
\hline
\end{tabular}

Notes. The last column lists integrated line ratios of $\mathrm{CO}(2 \rightarrow 1) / \mathrm{CO}(1 \rightarrow 0)$ with $\mathrm{CO}$ fluxes in $\mathrm{K} \mathrm{km} \mathrm{s}^{-1}$. To convert from Jy $\mathrm{km} \mathrm{s}^{-1}$ to W m${ }^{-2}$, the fluxes should be multiplied by $a=10^{-26} \frac{v_{0}}{c}$, where $a_{\mathrm{HI}}=4.74 \times 10^{-23}, a_{\mathrm{CO}(2 \rightarrow 1)}=7.69 \times 10^{-21}$ and $a_{\mathrm{CO}(1 \rightarrow 0)}=3.85 \times 10^{-21}$.

We present observations of [C II] emission in three regions of NGC 4214 using the GREAT instrument on board the SOFIA telescope. Our main objective is to extend the few studies of $\mathrm{CO}$-dark gas in galaxies by decomposing the spectrally resolved [C II] emission of NGC 4214 into fractions associated with the atomic and the molecular hydrogen gas content. In particular, our new observations allow us to determine which fraction of the $[\mathrm{C}$ II] emission can be associated spectrally with the COemitting gas and, in turn, which fraction is too far removed in velocity-space to be physically linked directly to the cold dense gas detected in CO. In Sect. 2 we describe the data, in Sect. 3 we describe our decomposition method with its results, and in Sect. 4 we present the associated gas fractions and masses. Our analysis is summarized and discussed in Sect. 5.

\section{Data}

\section{1. [C II] data}

\subsubsection{SOFIA data}

The [C II ] emission at $1900.54 \mathrm{GHz}(158 \mu \mathrm{m})$ was observed in NGC 4214 using the L2 band of the GREAT instrument (Heyminck et al. 2012) on board the airborne Stratospheric Observatory for Infrared Astronomy (SOFIA; Young et al. 2012). Observations were performed as part of observation cycle 2 in May 2014 and January 2015 with a half-power beam width of $14.1^{\prime \prime}(\sim 200 \mathrm{pc})$ and $1.16 \mathrm{~km} \mathrm{~s}^{-1}$ velocity resolution. Five positions in NGC 4214 were observed in the three main star-forming regions (Fig. 1; the coordinates can be found in Table 1). SOFIA has a pointing accuracy of $\sim 0.5^{\prime \prime}$. The data were processed with the eXtended bandwidth Fast Fourier Transform Spectrometer (XFFTS) and the standard GREAT calibrator (Guan et al. 2012). The data were calibrated to antenna temperature scale $\left(\eta_{\mathrm{f}}=\right.$ $0.97)$ and to the main beam temperature scale $\left(\eta_{\mathrm{mb}}=0.65-0.69\right.$ for L2). We converted the data from main beam temperature scale to Jansky using a conversion factor of $585 \mathrm{Jy} / \mathrm{K}$.

Before averaging the spectra, the baseline was removed using a polynomial of first order except in a few spectra from May 2014 where third-order polynomials were used. Data reduction was done using the CLASS software ${ }^{1}$. For further analysis we rebinned the data to $2.6 \mathrm{~km} \mathrm{~s}^{-1}$ resolution. The fluxes reported in Table 1 were calculated by direct integration of the rebinned spectra between 270 and $330 \mathrm{~km} \mathrm{~s}^{-1}$, except for region III where we used channels between 240 and $330 \mathrm{~km} \mathrm{~s}^{-1}$. These ranges were chosen based on the line widths also observed in $\mathrm{CO}$ and $\mathrm{HI}$.

\footnotetext{
1 http://www.iram. fr/IRAMFR/GILDAS
}

\subsubsection{Herschel/PACS}

NGC 4214 was also observed in the [C II] line with the PACS spectrometer (Poglitsch et al. 2010) on the Herschel Space Observatory (Pilbratt et al. 2010). The data were originally presented in Cormier et al. (2010) and reprocessed with the PACS spectrometer pipeline of the Herschel interactive processing environment (HIPE) v14.2. The PACS map covers a $1.6^{\prime} \times 1.6^{\prime}$ field of view with a spatial resolution of $12^{\prime \prime}$. Figure 1 shows the integrated intensity map observed with Herschel/PACS in the right panel. We compare the fluxes of GREAT and PACS for the regions observed with both instruments. To this end, the PACS map was convolved with a Gaussian kernel to the angular resolution of SOFIA/GREAT data and aperture photometry was applied to compute the fluxes shown in Table 1. For both [C II] datasets, the emission is strongest in pointing I-2 in the central region of NGC 4214 where the super star cluster is located and weakest in region III. The Herschel/PACS and SOFIA/GREAT fluxes are in relatively good agreement (within $40 \%$ ) in regions I and II. In region III, the GREAT flux is about two times higher than the PACS flux, but [C II] is not detected at high significance in this region.

\section{2. $[\mathrm{HI}]$ data}

NGC 4214 was observed in the $\mathrm{CO}(1 \rightarrow 0)$ transition line with the Caltech OVRO millimeter interferometer by Walter et al. (2001). The data have a velocity resolution of $1.30 \mathrm{~km} \mathrm{~s}^{-1}$ and the half-power beam width is $6.4^{\prime \prime} \times 5.7^{\prime \prime}$.

NGC 4214 was also included in the HERA CO-Line Extragalactic Survey (HERACLES; Leroy et al. 2009). The $\mathrm{CO}(2 \rightarrow 1)$ line at $230.54 \mathrm{GHz}(1.3 \mathrm{~mm})$ was observed with a half-power beam width of $13^{\prime \prime}$ and $2.6 \mathrm{~km} \mathrm{~s}^{-1}$ velocity resolution. Although the spectral resolution is higher for the OVRO $\mathrm{CO}(1 \rightarrow 0)$ data, we used $\mathrm{CO}(2 \rightarrow 1)$ to distinguish the origin of [C II] emission because the velocity range covered by the OVRO data is narrower, ranging only from 262 to $343 \mathrm{~km} \mathrm{~s}^{-1}$, while the HI emission is more extended than this. This can be seen in Fig. 2. A second disadvantage of using the OVRO interferometry data is that we might miss extended $\mathrm{CO}$ emission. However, in the low-metallicity environment of NGC 4214, we do not expect a significant amount of extended emission.

Nonetheless, we want to investigate the possibility of missing $\mathrm{CO}$ in the OVRO observations and thus calculate the flux ratios of $\mathrm{CO}(1 \rightarrow 0)$ and $\mathrm{CO}(2 \rightarrow 1)$. Table 1 provides the flux ratios (in $\mathrm{K} \mathrm{km} \mathrm{s}^{-1}$ ) for both OVRO and HERACLES datasets in each region. To calculate this quantity, we convolved the OVRO $\mathrm{CO}(1 \rightarrow 0)$ data to the angular resolution of the HERACLES observation and integrated by summation over the channels. 


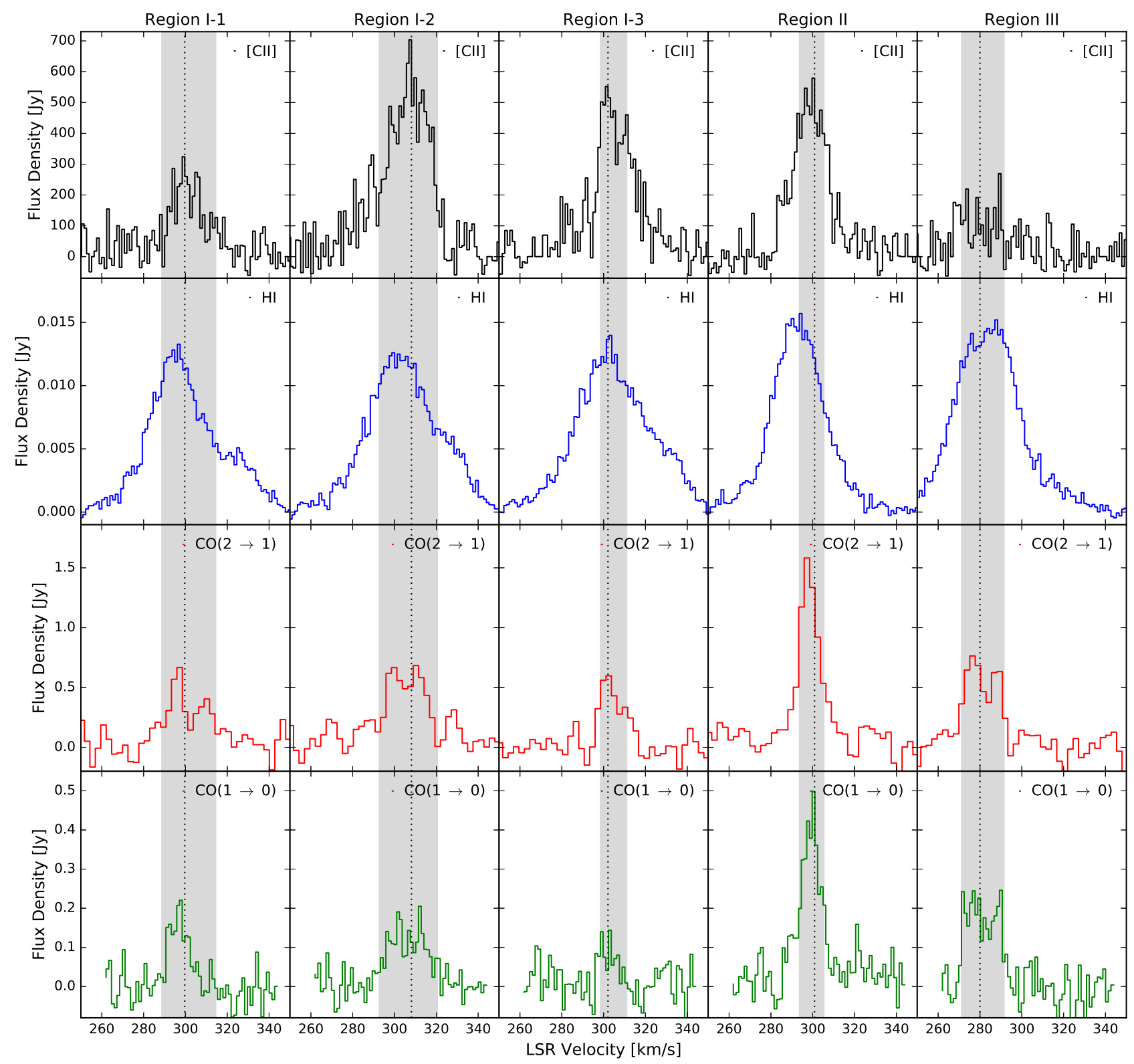

Fig. 2. Emission spectra of SOFIA/GREAT [C II] and ancillary CO and $\mathrm{H}$ in the five positions in NGC 4214. The spectra shown are based on the data that were spatially convolved with Gaussian kernels to the resolution of the SOFIA data (14"). The vertical lines indicate the channel with maximum [C II] emission, except for region III where it indicates the center of the broad profile. The gray-shaded areas indicate the FWHM ranges around the CO-peak positions. The original velocity resolutions shown here are $1.16 \mathrm{~km} \mathrm{~s}^{-1}, 1.29 \mathrm{~km} \mathrm{~s}^{-1}, 2.6 \mathrm{~km} \mathrm{~s}^{-1}$, and $1.30 \mathrm{~km} \mathrm{~s}$ - for [C II] $\mathrm{HI}, \mathrm{CO}(2 \rightarrow 1)$, and $\mathrm{CO}(1 \rightarrow 0)$, respectively.

Especially in region I, the values are higher than the typical ratios of 0.8 (Braine et al. 1993), but overall they agree within the uncertainties. The values seem reasonable, but we note that the OVRO data may be subject to missing flux (zero-spacing) so that the ratios formally represent an upper limit of the true ratio. However, given the strong radiation fields from ongoing star formation - in particular in regions I and II - and the lowmetallicity, we do not expect a diffuse CO component.

We used HI data from The HI Nearby Galaxy Survey (THINGS; Walter et al. 2008) which performed observations of the atomic hydrogen content in nearby galaxies using the $21 \mathrm{~cm}$ $(1.4204 \mathrm{GHz})$ line of atomic hydrogen with a half-power beam width of $14.69^{\prime \prime} \times 13.87^{\prime \prime}$ and $1.29 \mathrm{~km} \mathrm{~s}^{-1}$ spectral resolution.

Table 1 lists the fluxes for $\mathrm{HI}, \mathrm{CO}(2 \rightarrow 1)$, and $\mathrm{CO}(1 \rightarrow$ $0)$ in all regions. The fluxes were derived after convolving the $\mathrm{CO}$ data to the angular resolution of SOFIA/GREAT. For the
CO lines, we chose channels between 270 and $330 \mathrm{~km} \mathrm{~s}^{-1}$ for integration and for $\mathrm{HI}$ we take the channels between 250 and $350 \mathrm{~km} \mathrm{~s}^{-1}$. In region III, however, we set the integration range to $240-300 \mathrm{~km} \mathrm{~s}^{-1}$ for both CO and H I spectra. The H I fluxes are almost constant over the regions, while the $\mathrm{CO}$ fluxes have their maximum in region II.

\subsection{Description of the spectra}

The spectra of [C II], $\mathrm{HI}, \mathrm{CO}(2 \rightarrow 1)$, and $\mathrm{CO}(1 \rightarrow 0)$ in the five regions observed by SOFIA/GREAT are shown in Fig. 2.

By fitting Gaussians, we find that the [C II] emission lines have widths of $\sim 20 \mathrm{~km} \mathrm{~s}^{-1}$ and are centered at about $300 \mathrm{~km} \mathrm{~s}^{-1}$, except in region III where the data show weak [C II] emission 
Table 2. Parameters of the Gaussian fitting of the $\mathrm{HI}$ and $\mathrm{CO}$ spectra.

\begin{tabular}{lcccccc}
\hline \hline Region & $\begin{array}{c}\mathrm{HI} \\
p_{\mathrm{HI}, 1} \\
{\left[\mathrm{~km} \mathrm{~s}^{-1}\right]}\end{array}$ & $\begin{array}{c}F W H M_{\mathrm{HI}, 1} \\
{\left[\mathrm{~km} \mathrm{~s}^{-1}\right]}\end{array}$ & $\begin{array}{c}p_{\mathrm{HI}, 2} \\
{\left[\mathrm{~km} \mathrm{~s}^{-1}\right]}\end{array}$ & $\begin{array}{c}F W H M_{\mathrm{HI}, 2} \\
{\left[\mathrm{~km} \mathrm{~s}^{-1}\right]}\end{array}$ & $\begin{array}{c}\mathrm{CO}(2 \rightarrow 1) \\
p_{\mathrm{CO}} \\
{\left[\mathrm{km} \mathrm{s}^{-1}\right]}\end{array}$ & $\begin{array}{c}F W H M_{\mathrm{CO}} \\
{\left[\mathrm{km} \mathrm{s}^{-1}\right]}\end{array}$ \\
\hline I-1 & 295.7 & 29.1 & 327.1 & 21.5 & 301.6 & 25.8 \\
I-2 & 302.7 & 35.0 & 331.4 & 15.5 & 306.6 & 27.8 \\
I-3 & 302.7 & 36.2 & 333.4 & 18.7 & 304.8 & 12.5 \\
II & 293.6 & 28.7 & - & - & 299.5 & 11.7 \\
III & 284.0 & 35.3 & - & - & 281.4 & 20.2 \\
\hline
\end{tabular}

Notes. Peak positions $p$ and FWHMs $(=2.35 \sigma)$. Two Gaussians were fitted to the H I spectra of the pointings in region I.

centered at lower velocities of $\sim 280 \mathrm{~km} \mathrm{~s}^{-1}$ with widths of $\sim 30 \mathrm{~km} \mathrm{~s}^{-1}$.

The H I profiles are broader (about $30 \mathrm{~km} \mathrm{~s}^{-1}$ ). Again, in region III the peak position is at lower velocities, but in regions I-1, I-2, and II we also find the peak position shifted to slightly lower velocities compared to the [C II] spectra.

We note that the $\mathrm{CO}$ emission in both transition lines is clearly strongest in region II with a width of $\sim 15 \mathrm{~km} \mathrm{~s}^{-1}$, while the other regions show widths between 20 and $25 \mathrm{~km} \mathrm{~s}^{-1}$. The spectra peak at $\sim 300 \mathrm{~km} \mathrm{~s}^{-1}$ except for region III where the velocity is again lower. The spectra of $\mathrm{CO}(2 \rightarrow 1)$ and $\mathrm{CO}(1 \rightarrow 0)$ have similar shapes. However, in regions I-1, I-2, and I-3 there are some features to the right and left of the central peak that are only visible in one of the two datasets.

Table 1 shows that the H I fluxes are almost constant throughout the five regions, as is also indicated by the spectra. We find that the $\mathrm{CO}$ emission is weak compared to [C II] with flux ratios of $[\mathrm{C} \mathrm{II}] / \mathrm{CO}(1 \rightarrow 0)$ between $2 \times 10^{4}$ and $17 \times 10^{4}$ with a clear minimum in region III and the maximum in region I-2 (see also Cormier et al. 2010).

\section{Decomposition of [CII] spectra}

The main goal of this work is to determine how much of the [C II] emission traces the dense molecular material that is also probed by $\mathrm{CO}$. If it were a one-to-one tracer, the velocity profiles of [C II] and $\mathrm{CO}$ would be similar. However, as Fig. 2 already shows, the [C II] profiles in NGC 4214 show broader wings that are not seen in CO. To determine the maximum amount of [C II] that can be associated with the molecular phase and to investigate the origin of these wings, we decompose the spectrally resolved [C II] observations into contributions correlated with $\mathrm{H}_{2}$ (as traced by $\mathrm{CO}(2 \rightarrow 1)$ ) and $\mathrm{HI}$. In doing so, we assume that $\mathrm{CO}(2 \rightarrow 1)$ and $\mathrm{HI}$ trace material with uniform conditions. The consideration of atomic gas with different conditions is discussed in Sect. 5.1.2.

We decompose our [C II] spectra into a linear combination of $\mathrm{HI}$ and $\mathrm{CO}$ in two different ways. The first approach (channel method) uses the $\mathrm{CO}$ and $\mathrm{H}$ I spectra directly by adding them manually (see Appendix A). The second approach uses Gaussian fits for the decomposition, as described in the following. Although neither method shows striking inconsistencies, we focus on the Gaussian fit method as it appears to be more robust.

\subsection{Description of the Gaussian fit method}

We use Gaussian fits to decompose the [C II] spectra. In this approach, we first fit $\mathrm{HI}$ and $\mathrm{CO}(2 \rightarrow 1)$ spectra independently with Gaussians. We fit two independent Gaussian curves to the
H I spectra in region I as there seem to be two distinct components (see Sect. 2). For $\mathrm{CO}(2 \rightarrow 1)$, we only use one component because of the lower signal-to-noise ratio. Table 2 lists the fitted peak positions and widths (FWHM of the Gaussian curves) for the HI and CO spectra in the five regions in NGC 4214. Figure 3 shows that two Gaussians reproduce the H I data well in the pointings of region I; however, the parameters of the second Gaussian are less well constrained. We note that the first component of $\mathrm{HI}$ in region I has the same width as the H I spectra in region II and III indicating that the second component might be emission on the line of sight not connected to the star-forming region. The $\mathrm{CO}(2 \rightarrow 1)$ spectrum in region I-1 is fitted with a very broad Gaussian $\left(F W H M \approx 26 \mathrm{~km} \mathrm{~s}^{-1}\right)$, whereas the $\mathrm{CO}(1 \rightarrow 0)$ is fitted with a FWHM of $\approx 11 \mathrm{~km} \mathrm{~s}^{-1}$. We approximate the $\mathrm{CO}$ width in region $\mathrm{I}-1$ for the latter analysis to be $18 \mathrm{~km} \mathrm{~s}^{-1}$, the average FWHM of the original fitting of the $\mathrm{CO}(2 \rightarrow 1)$ and $\mathrm{CO}(1 \rightarrow 0)$ spectra. We discuss how changing the FWHM in this region to the individually fitted values $\left(11\right.$ and $\left.26 \mathrm{~km} \mathrm{~s}^{-1}\right)$ affects the decomposition results in the following section. In the other regions fitting the $\mathrm{CO}(1 \rightarrow 0)$ and $\mathrm{CO}(2 \rightarrow 1)$ spectra yields comparable widths.

We then normalize the $[\mathrm{C} \mathrm{II}]$ spectra to their maxima and fit them with Gaussians of the form

$$
\begin{aligned}
g(v)= & A_{1} \exp \left(-\frac{\left(v-p_{\mathrm{HI}, 1}\right)^{2}}{2 \sigma_{\mathrm{HI}, 1}^{2}}\right)+A_{2} \exp \left(-\frac{\left(v-p_{\mathrm{HI}, 2}\right)^{2}}{2 \sigma_{\mathrm{HI}, 2}^{2}}\right) \\
& +B \exp \left(-\frac{\left(\mathrm{v}-p_{\mathrm{CO}}\right)^{2}}{2 \sigma_{\mathrm{CO}}^{2}}\right) .
\end{aligned}
$$

The peak positions $p$ and widths $\sigma$ are taken as fixed parameters determined by the above-mentioned fits. The coefficients $A_{i}$ (H I amplitudes) and $B(\mathrm{CO})$ are used as free parameters for the fit. In region II and III, we use only one H I term. In region III, we rebin the $\left[\mathrm{C}\right.$ II] spectrum to a resolution of $5 \mathrm{~km} \mathrm{~s}^{-1}$ to improve the signal-to-noise ratio.

\subsection{Results}

Figure 4 shows the [C II] spectra in black with the best fitting curves overlaid in red. The fitted amplitudes and their uncertainties are given in Table 3. The stated uncertainties are the $1 \sigma$ errors from the fitting procedure. In the three pointings of region I, we find that the best fit is provided when the contribution of the second HI component is close to zero. This supports our assumption that this second component may not be connected to the star-forming region while the $[\mathrm{C}$ II $]$ emission is.

In addition, we note that the $\mathrm{CO}$ amplitudes are higher than the sum of $\mathrm{HI}$ amplitudes in all regions except for region III, where the uncertainties are high. The [C II] spectra are best decomposed when using large fractions of the $\mathrm{CO}(2 \rightarrow 1)$ spectra 

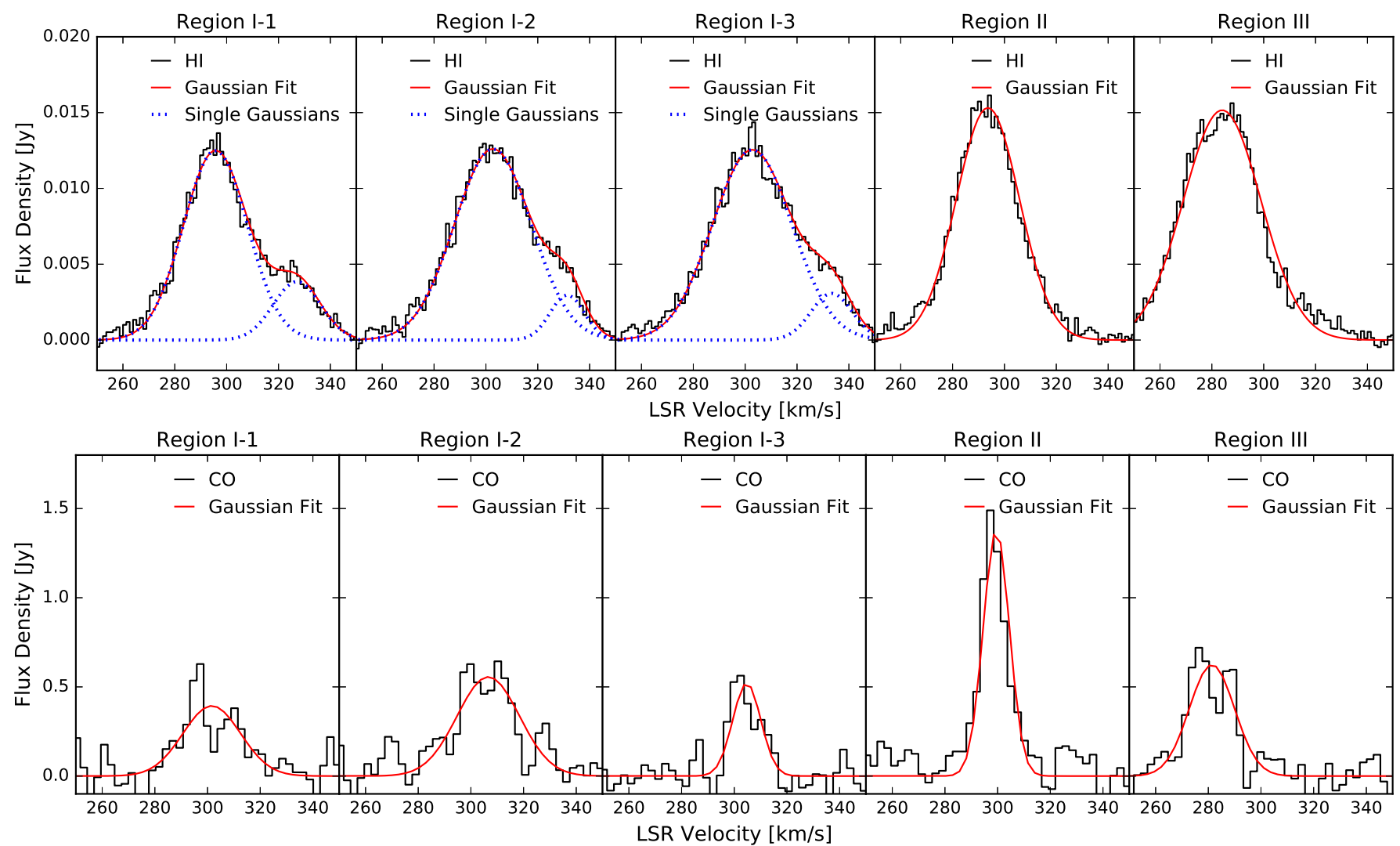

Fig. 3. Upper row: H I spectra of the five pointings in NGC 4214 fitted with Gaussian curves. For the spectra in region I, we use two Gaussians (single components in blue). Bottom row: $\mathrm{CO}(2 \rightarrow 1)$ fitted with single Gaussian curves.

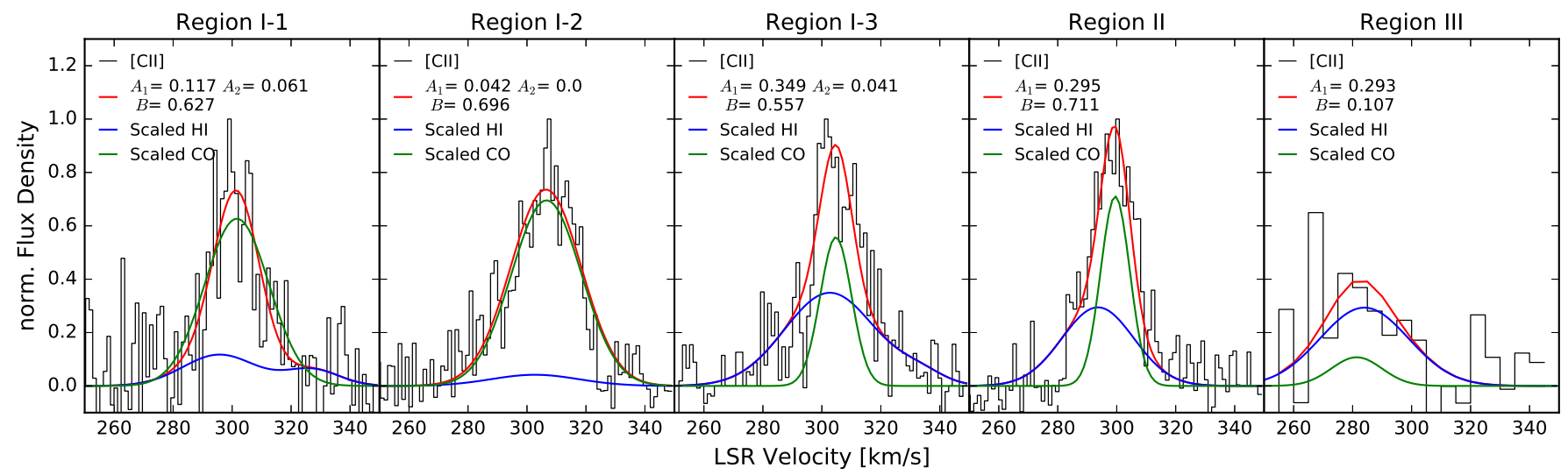

Fig. 4. Gaussian fit method. [C II] spectra in the five pointings fitted with Gaussian curves as described by Eq. (1). In region II and III we only fit two Gaussian curves, but in the pointings of region I we use two H I components. The [C II] spectra in region III was rebinned to $5 \mathrm{~km} \mathrm{~s}^{-1}$.

Table 3. Results from the Gaussian fit decomposition.

\begin{tabular}{lccccc}
\hline \hline Region & $A_{1}$ & $A_{2}$ & $B$ & $I_{[\mathrm{C} \text { II }}(\mathrm{CO}) / I_{[\mathrm{C} \mathrm{II}]}$ & \\
& $\mathrm{H} \mathrm{I}$ & $\mathrm{H} \mathrm{I}$ & $\mathrm{CO}$ & central value & $1 \sigma$ interval \\
\hline I-1 & $0.12 \pm 0.08$ & $0.06 \pm 0.05$ & $0.62 \pm 0.10$ & $77.4 \%$ & $65-85 \%$ \\
I-2 & $0.04 \pm 0.10$ & $0.00 \pm 0.04$ & $0.70 \pm 0.11$ & $93.0 \%$ & $85-100 \%$ \\
I-3 & $0.35 \pm 0.04$ & $0.04 \pm 0.04$ & $0.56 \pm 0.08$ & $34.3 \%$ & $25-45 \%$ \\
II & $0.29 \pm 0.04$ & - & $0.71 \pm 0.06$ & $49.5 \%$ & $40-60 \%$ \\
III & $0.29 \pm 0.11$ & - & $0.18 \pm 0.24$ & $17.5 \%$ & $0-30 \%$ \\
\hline
\end{tabular}

Notes. $A_{1}, A_{2}$ and $B$ are the coefficients of the components (Eq. (1)). Columns 5 and 6 list the central values and $1 \sigma$ intervals of the fraction of $[\mathrm{C} \mathrm{II}]$ intensity associated with $\mathrm{CO}$ (see Sect. 3.2). The intensity fractions associated with $\mathrm{H}$ I are the remaining fraction of the [C II] intensity. 
in agreement with the scaling coefficients used in the channel method (Appendix A). To verify that the Gaussian fits recover the [C II] fluxes, we compare the velocity integrated fluxes of the original [C II] data with the combined Gaussians using the same velocity ranges as before. The ratios of Gaussians and direct integration are very close to unity and therefore our fits recover all of the [C II] emission.

With the spectral decomposition of [C II] emission determined in this way, we calculate the fractions of [C II] intensity associated with $\mathrm{CO}(2 \rightarrow 1)$ and $\mathrm{HI}$. Each fraction is determined as the ratio between the integrated [C II]-fitted profiles and the accordingly scaled $\mathrm{HI}$ and $\mathrm{CO}$ spectra that were used in the decomposition. Simply put, we compare the areas below the red curves (final fit) in Fig. 4 to the areas below the green and blue curves (corresponding $\mathrm{H} \mathrm{I}$ and $\mathrm{CO}$ contributions to the final fit). The $[\mathrm{C} \mathrm{II}]$ intensity fractions associated with $\mathrm{CO}(2 \rightarrow 1)$ are listed in Table 3. In region I-2, we find almost no contribution from the $\mathrm{HI}$, and the $\mathrm{CO}(2 \rightarrow 1)$ contribution to the [C II] flux is also higher than the $\mathrm{HI}$ contribution in region I-1. In region II, the parts are almost equally split. In region III, we can only associate a rather small fraction of $\mathrm{CO}$ emission with the [C II].

We validate the uncertainties on the amplitudes given by the IDL routine GAUSSFIT in the following manner. We vary the $\mathrm{CO}$ amplitude from 0.3 to 0.9 in steps of 0.1 and re-fit the $\mathrm{H} \mathrm{I}$ amplitude. For each chosen CO amplitude, we estimate the goodness of the fit by considering the residuals (signal - fit) normalized by the noise level of the [C II] data. We calculate the $\chi^{2}$ by summing these residuals over the channels between 280 and $320 \mathrm{~km} \mathrm{~s}^{-1}$. The $1 \sigma$ interval that we find corresponds well with the uncertainties given by the fitting procedure. This range of amplitudes is then translated into uncertainties on the intensity fractions (Col. 5 of Table 3 ). We find that these uncertainties are about $\pm 10 \%$, except for region III where they are at least $\pm 20 \%$. For region I-1, we also explore the sensitivity of the results to the value of the CO FWHM because the two available CO lines yield substantially different values. When using $26 \mathrm{~km} \mathrm{~s}^{-1}$, i.e., the value derived from the $\mathrm{CO}(2 \rightarrow 1)$, we obtain a poor fitting result because the $\mathrm{CO}(2 \rightarrow 1)$ line is broader than the observed [C II] line. As a result, the wings and the peak in the [C II] cannot be reproduced at the same time. When using the FWHM determined from $\mathrm{CO}(1 \rightarrow 0)$, we obtain a reasonable decomposition with a $\mathrm{CO}$ amplitude of 0.55 and a residual comparable to when using the average FWHM. This results in a CO-associated intensity fraction of $(56 \pm 10) \%$, a value around $20 \%$ lower than that given in Table 3 calculated with the average FWHM $\left(18 \mathrm{~km} \mathrm{~s}^{-1}\right)$. We note that the maximum $\mathrm{CO}$ percentage provides a hard limit on the amount of $[\mathrm{CII}]$ that can be associated with $\mathrm{CO}$. The lower limit is less strict because it depends on the nature of the H I-emitting gas (see Sect. 5.1.2). The H I profiles might contain narrow and broad components that we cannot separate owing to the limited spatial resolution of the H I observations.

The quality of the input spectra that define the Gaussian components plays an important role in our method. The uncertainties of our results could be further reduced with deeper CO observations. This is particularly true for region I-1, but is true for the other regions as well.

\section{Gas masses from different tracers}

\section{1. $\mathrm{C}^{+}$column densities and associated gas masses}

The decomposition described in the previous section divides the $[\mathrm{C} \mathrm{II}]$ emission into fractions associated with $\mathrm{HI}$ and $\mathrm{CO}(2 \rightarrow 1)$ emission. We find that the $\mathrm{CO}$ fraction tends to be larger; however, the [C II] profiles show both narrow and broad components. Assuming different sets of conditions in the ISM, we calculate the gas masses that these components would correspond to. In principle, at our spatial resolution $\left(14^{\prime \prime} \sim 200 \mathrm{pc}\right)$, the ISM should contain ionized gas, cold neutral medium (CNM), warm neutral medium (WNM), and dense neutral/molecular gas (PDR). While the narrow components in the [C II] profiles can be associated with the PDR, the broad components can correspond to ionized gas, WNM, and/or CNM.

We neglect the contribution from ionized gas because of a previous study of NGC 4214 by Dimaratos et al. (2015). They perfomed a self-consistent radiative transfer modeling of the H II gas and PDR in regions I and II of NGC 4214 using a suite of mid-IR and far-IR lines. They find that the H II region models contribute only a small percent of the [C II] emission. Using the [N II] lines at $122 \mu \mathrm{m}$ and $205 \mu \mathrm{m}$, they also estimate the contribution of a diffuse ionized phase to the [C II] emission to be less then $8 \%$.

We study the extended component, i.e., the HI-associated [C II] flux, as well as the CO-matched dense material by calculating gas masses (corresponding to $\mathrm{H}$ I or $\mathrm{H}_{2}$ masses) assuming different sets of ISM conditions (see Table 4). None of the quoted masses includes helium.

We calculate $\mathrm{C}^{+}$column densities from the observed intensity $I_{[\mathrm{CII}]}$ following Madden et al. (1997):

$$
\begin{aligned}
N\left(\mathrm{C}^{+}\right)= & 1.06 \times 10^{15} \frac{\int F_{\nu} \mathrm{d} v\left[\mathrm{Jy} \mathrm{km} \mathrm{s}^{-1}\right]}{\Theta\left[{ }^{\prime \prime}\right]^{2}} \\
& \times\left(\frac{1+2 \exp (-91.3 \mathrm{~K} / T)+n_{\text {crit }} / n}{2 \exp (-91.3 \mathrm{~K} / T)}\right) .
\end{aligned}
$$

Here $T$ is the kinetic gas temperature in Kelvin, and $n$ the density and $n_{\text {crit }}$ the critical density of the main collision partner of $\mathrm{C}^{+}$ both in $\mathrm{cm}^{-3}$. We assume that all of the carbon is ionized in the CNM, WNM, and in the PDR, and that the [C II] line is excited by collisions with atomic hydrogen in the phases associated with H I emission and by collisions with molecular hydrogen in the PDR (see Table 4). We use the intensity fractions from Table 3 to scale the column density according to the ISM phase that is considered.

For the abundance of ionized carbon, $X_{\mathrm{C}^{+} / \mathrm{H}}=5.07 \times 10^{-5}$ is used in region I and $X_{\mathrm{C}^{+} / \mathrm{H}}=7.24 \times 10^{-5}$ is used in region II (Kobulnicky \& Skillman 1996). There is no measurement for region III, so we used the lower value since this region is thought to be less evolved.

We then calculate the gas masses traced by [C II] and associated with $\mathrm{CO}$ and $\mathrm{HI}$ by adapting Eq. (3) from Pérez-Beaupuits et al. (2015) to our data. We assume a beam filling factor of 1 :

$$
M_{\mathrm{gas}}\left[M_{\odot}\right]=3.16 \times 10^{-16} \frac{N\left(\mathrm{C}^{+}\right)}{X_{\mathrm{C}^{+} / \mathrm{H}}}
$$

The resulting gas masses are reported in Table 4 using intensity fractions with uncertainties from Table 3 . We emphasize that these masses are theoretical values assuming that the $\mathrm{H}$ I and $\mathrm{CO}$-associated fractions of the $[\mathrm{C} \mathrm{II}]$ intensity originate from regions of uniform ISM conditions. We discuss these model values further in Sect. 5. The gas masses are sensitive to the assumed ISM conditions. For example, changing the CNM temperature from 80 to $100 \mathrm{~K}$ results in CNM-associated gas masses that are reduced by $20 \%$ compared to the values given in Table 4 . By changing the electron density from 100 to $200 \mathrm{~cm}^{-3}$, the gas mass is about half of the reported value. Similarly, changing the 
Table 4. Assumed sets of conditions for ISM phases used to calculate [C II]-associated gas masses, and observed $\mathrm{H}_{\mathrm{I}}$ and $\mathrm{H}_{2}$ masses.

\begin{tabular}{c|ccc}
\hline \hline & CNM & WNM & PDR CO \\
\hline Collision partner of $\mathrm{C}^{+}$ & $\mathrm{H}$ & $\mathrm{H}$ & $\mathrm{H}_{2}$ \\
$T[\mathrm{~K}]$ & 80 & 8000 & 150 \\
$n\left[\mathrm{~cm}^{-3}\right]$ & 100 & 0.5 & $10^{4}$ \\
$n_{\text {crit }}\left[\mathrm{cm}^{-3}\right]$ & 3000 & 1600 & 6100 \\
\hline
\end{tabular}

\begin{tabular}{ccc|ccc}
\hline \hline Region & $\begin{array}{c}M_{\mathrm{HI}} \\
{\left[10^{5} M_{\odot}\right]}\end{array}$ & $\begin{array}{c}M_{\mathrm{H}_{2}} \\
{\left[10^{5} M_{\odot}\right]}\end{array}$ & $\begin{array}{c}M_{\mathrm{gas}}\left([\mathrm{C} \mathrm{II}]_{\mathrm{HI}, \mathrm{CNM}}\right) \\
{\left[10^{5} M_{\odot}\right]}\end{array}$ & $\begin{array}{c}M_{\mathrm{gas}}\left([\mathrm{C} \mathrm{II}]_{\mathrm{HI}, \mathrm{WNM}}\right) \\
{\left[10^{7} M_{\odot}\right]}\end{array}$ & $\begin{array}{c}M_{\mathrm{gas}}\left([\mathrm{C} \mathrm{III}]_{\mathrm{CO}, \mathrm{PDR}}\right) \\
{\left[10^{5} \mathrm{M}_{\odot}\right]}\end{array}$ \\
\hline I-1 & 9.30 & 1.94 & $23.16_{-9.37}^{+9.01}$ & $7.57_{-3.07}^{+2.95}$ & $6.88_{-0.90}^{+0.94}$ \\
I-2 & 10.11 & 3.12 & $4.25_{-4.25}^{+20.73}$ & $1.39_{-1.39}^{+6.78}$ & $24.58_{-2.08}^{+0.43}$ \\
I-3 & 10.62 & 1.27 & $117.39_{-23.03}^{+14.72}$ & $38.40_{-7.53}^{+4.82}$ & $7.14_{-1.47}^{+2.30}$ \\
II & 9.36 & 3.65 & $60.36_{-10.46}^{+14.47}$ & $19.74_{-3.43}^{+4.73}$ & $6.44_{-1.45}^{+1.05}$ \\
III & 11.20 & 2.42 & $53.33_{-8.08}^{+11.31}$ & $17.44_{-2.64}^{+3.70}$ & $1.13_{-1.13}^{+0.81}$ \\
\hline
\end{tabular}

Notes. We present the observed $\mathrm{H}$ I and $\mathrm{H}_{2}$ masses and compare them to theoretical values of the gas mass calculated from the $\mathrm{H} \mathrm{I}_{\mathrm{I}}$ - or $\mathrm{H}_{2}$-associated components of the $[\mathrm{C} \mathrm{II}]$ intensities under the assumption that all of the $\mathrm{HI}$ - or CO-associated [C II] emits from an ISM phase with the conditions stated above. The uncertainties result from the uncertainties given in Table 3. PDR conditions are from Dimaratos et al. (2015), critical densities from Goldsmith et al. (2012).

PDR temperature to $100 \mathrm{~K}$, the theoretical mass is reduced by $18 \%$. By decreasing the assumed PDR density by a factor of 2 to $5000 \mathrm{~cm}^{-3}$, the resulting mass is reduced by $20 \%$. However, owing to the inherently high density in this phase, the masses are less sensitive to an increase in the density. If the density is increased to $10^{5} \mathrm{~cm}^{-3}$, the mass increases by $25 \%$. We discuss the sensitivity to the assumed parameters further in Sect. 5.1.

\subsection{Gas masses traced by $\mathrm{HI}$ and $\mathrm{CO}$}

In addition to the gas masses traced by [C II], we also calculate the atomic and molecular gas masses using the $\mathrm{HI}$ and $\mathrm{CO}(1 \rightarrow 0)$ intensities directly (Table 4$)$.

The H I mass is calculated using the relation

$$
M_{\mathrm{HI}}\left[M_{\odot}\right]=2.36 \times 10^{5}(D[\mathrm{Mpc}])^{2} \int F_{\nu} \mathrm{d} v\left[\mathrm{Jy} \mathrm{km} \mathrm{s}^{-1}\right]
$$

To calculate the molecular gas mass from $\mathrm{CO}(1 \rightarrow 0)$ emission, we use Eq. (2) from Bolatto et al. (2013)

$$
M_{\mathrm{H}_{2}}\left[M_{\odot}\right]=\frac{\alpha_{\mathrm{CO}}}{1.36} L_{\mathrm{CO}(1 \rightarrow 0)},
$$

where $\alpha_{\mathrm{CO}}=4.3 M_{\odot}\left(\mathrm{K} \mathrm{km} \mathrm{s}^{-1} \mathrm{pc}^{2}\right)^{-1}$ is the Galactic value of the CO-to- $\mathrm{H}_{2}$ conversion factor and $L_{\mathrm{CO}(1 \rightarrow 0)}$ is expressed in $\mathrm{K} \mathrm{km} \mathrm{s}^{-1} \mathrm{pc}^{2}$. The factor of 1.36 is the helium contribution that we do not consider here. Table 4 lists the $\mathrm{HI}$ and $\mathrm{H}_{2}$ masses calculated from $\mathrm{H}$ I and $\mathrm{CO}$ emission directly.

While the H I mass distribution is almost constant throughout the regions, the values for $\mathrm{H}_{2}$ masses differ from region to region by up to a factor of 2 ; the maximum is found in region II. We find ratios of $M_{\mathrm{H}_{2}}$ to $M_{\mathrm{HI}}$ of $\sim 0.2$. These ratios are higher than the 0.02 found by Leroy et al. (2009) for the whole galaxy because we only study the star-forming regions and therefore only include small fractions of the extended $\mathrm{HI}$.

\section{Discussion}

\section{1. [C II] emission from different ISM phases}

We showed in our analysis that the [C II] velocity profiles do not have the same shapes as the $\mathrm{CO}$ profiles, but they show broad wings that can be reproduced by $\mathrm{HI}$. We want to understand what this means in terms of how reliably [C II] traces the molecular phase and what the origin of these broad wings might be. With our decomposition and the calculated model gas masses (Table 4), we can explore this issue.

\subsubsection{Molecular gas}

The [C II] gas mass associated with the dense PDR (traced by $\mathrm{CO}$ emission) reaches its maximum in region I-2 and its minimum in region III. In the other regions the mass is almost constant. Except for region III, we find that the [C II] emission traces 3-8 times more mass than CO.

We can estimate how much of the molecular mass is CO-dark by comparing the molecular gas mass found with [C II] emission assuming PDR conditions with the molecular gas mass directly determined from $\mathrm{CO}(1 \rightarrow 0)$ observations (Cols. 6 and 3 in Table 4, respectively). We calculate the CO-dark molecular mass fraction as the molecular mass derived from [C II] divided by the total molecular mass (from $\mathrm{CO}$ and [C II]). In regions I-1, I-2, and I-3, the fraction of total molecular gas mass only seen by [C II] is high with $(78 \pm 13) \%,(89 \pm 10) \%$, and $\left(85_{-25}^{+15}\right) \%$, respectively, suggesting a large amount of CO-dark$\mathrm{H}_{2}$ gas extended over region $\mathrm{I}$ as is expected in low-metallicity environments. The errors quoted here result from the $1 \sigma$ uncertainties in the decomposition. Since Maíz-Apellániz et al. (1999) and MacKenty et al. (2000) found that region I is resolved into two different regions around two massive star clusters, it is not surprising that we find different fractions in the three pointings. We note, that region I-2 covers the super star cluster (cluster A, see Maíz-Apellániz et al. 1999; or Maíz-Apellániz 2001), while a second cluster (labeled B) is covered by pointing I-3. While region I has an age of 3-4 Myr (Úbeda et al. 2007b), region II is a more compact and younger ( $2 \mathrm{Myr})$ star-forming region with 
higher metallicity (by $\sim 0.1$ dex; Kobulnicky \& Skillman 1996). In region II, we report that $(64 \pm 20) \%$ of the total molecular mass is found with [C II] emission. This value is lower than the those found in region I and might be explained by increased dust shielding, as indicated by the slightly higher metallicity and less porous ISM structure of this younger region. On average, around $79 \%$ of the total molecular mass is CO-dark.

The gas mass found in $\mathrm{CO}$ slightly exceeds the mass found by $[\mathrm{C}$ II] in region III. We can therefore make no statement about the CO-dark gas in this region owing to a combination of an uncertain decomposition and low [C II] intensity. Region III is the least evolved region with no visible star formation.

We note that the $[\mathrm{C} \mathrm{II}]$-associated gas masses vary with the assumed conditions. While small deviations from the assumed ISM conditions could reduce the CO-dark fractions, we find average PDR densities of $\sim 500 \mathrm{~cm}^{-3}$ or temperatures of $\sim 35 \mathrm{~K}$ are needed to explain the observed $\mathrm{H}_{2}$ masses without any $\mathrm{CO}$-dark gas. Those conditions are not in agreement with the PDR analysis of Dimaratos et al. (2015). These are strong arguments in favor of a massive $\mathrm{CO}$-dark gas phase.

Instead of masses, we can also quote fractions of total [C II] intensity assigned to the CO-dark phase. In region II, we can assign $(33 \pm 9) \%$ of the total [C II] intensity to this phase. In regions I-1, I-2, and I-3 we find values of $(58 \pm 11) \%$, $(87 \pm$ $12) \%$, and $(32 \pm 12) \%$, respectively.

\subsubsection{Atomic gas}

For the atomic gas, we have estimated gas masses assuming WNM and CNM conditions. In the WNM case, very large masses, on the order of $10^{8} M_{\odot}$, would be required in order to explain the observed [C II] emission. Given that these are much larger than the observed H I masses from the $21 \mathrm{~cm}$ line, only $\sim 1 \%$ of the observed [C II] intensity can arise from the WNM. In their study of the Milky Way, Pineda et al. (2014) also find no significant contribution of the WNM to the [C II] emission.

Nonetheless, the WNM can make up a significant fraction of the total atomic content (e.g., Kulkarni \& Heiles 1987; Heiles \& Troland 2003), especially at large velocities due to high temperatures. The widths of the H I profiles that we observe could therefore be caused by the WNM. In principle, it might be better to first subtract any WNM contribution to the $\mathrm{H}$ I line as the [C II] line is less sensitive to this warm phase. However, it is not easy to determine what causes the broad H I profile. Sellwood \& Balbus (1999) and Tamburro et al. (2009) argue that turbulence stirred by magnetorotational instability can also lead to broad line widths. Moreover, there could also be significant substructure within the telescope beam. To constrain any contribution from the WNM, further H I observations with higher spatial resolution would be needed.

Even if we assume that the $\mathrm{HI}$-associated [C II] originates from the CNM, the corresponding masses exceed the upper limits given by the $\mathrm{H}$ I observations directly. We find that between 4 and $10 \%$ of our total [C II] intensity can arise from the CNM in order to reproduce the $\mathrm{HI} 21 \mathrm{~cm}$ masses. In region III, we can assign $(17 \pm 6) \%$ of the [C II] emission to the CNM. In a study of the metal-poor irregular galaxy IC10, Madden et al. (1997) assigned about $10 \%$ of [C II] emission to the CNM (and $~ 10 \%$ to the ionized medium) in agreement with our results. This indicates that the $\mathrm{HI}$-associated [C II] not only emits from the CNM, but also from a denser phase. To match the H I masses, we would need densities of about $1000 \mathrm{~cm}^{-3}$ on average when assuming temperatures of $80 \mathrm{~K}$ and a critical density of $3000 \mathrm{~cm}^{-3}$. We note that it is not realistic that all the $\mathrm{HI}$-associated [C II] emits under these conditions, but it indicates that our CNM is not dense enough to be the sole origin of the broad wing component in the $[\mathrm{C}$ II] profiles. Furthermore, such high densities are unlikely as they would presumably appear as narrow wings, and not the broad wings that are seen in the [C II]. The true nature of these wings is not clearly understood.

Parts of the H I-associated [C II] might come from the same dense PDR regions that are also traced by $\mathrm{CO}$. This dense component of the H I profile should have a similar velocity profile to $\mathrm{CO}$, and as Fig. 2 shows, it would be possible to fit a dense $\mathrm{CO}$-associated component to the H I near the peak. Therefore, when using only the three tracers discussed in this work, it becomes difficult to separate dense and extended components. Our findings show that further studies with additional, clearly separable tracers of molecular and atomic gas phases would be needed to resolve this issue.

\subsection{Comparison with other sources}

Previous studies on star-forming regions and ISM conditions in nearby galaxies have shown a complex origin of [C II] emission. Requena-Torres et al. (2016) performed a study of carbon gas with high spatial and velocity resolution in low-metallicity star-forming regions $\mathrm{N} 66, \mathrm{~N} 25+26$, and $\mathrm{N} 88$ of the Small Magellanic Cloud (SMC). While N 66 is an extended (100-150 pc) gas cloud with bright core, N 25 and N 26 are part of a group of $\mathrm{H}$ II regions and $\mathrm{N} 88$ is compact ( $\sim 1 \mathrm{pc})$ source in the SMC wing. They found [C II] emission profiles that are up to $50 \%$ wider in velocity than the corresponding $\mathrm{CO}$ profiles. They suggest that the gas traced with $\mathrm{CO}$ is embedded in a larger molecular cloud only seen by [C II] in the observed region. From studies on the $\mathrm{C}^{+}$column densities they concluded that most of the [C II] emission originates from CO-dark molecular gas. Only a few percent are contributed by either ionized and atomic hydrogen. The transition lines of CO only trace between 5 an $40 \%$ percent of the molecular gas found in the observed regions.

Like the SMC, the LMC provides spatially and velocityresolved observations of low-metallicity environments. Okada et al. (2015) studied the star-forming region $\mathrm{N} 159$ in the LMC with [C II] SOFIA/GREAT observations in comparison with different $\mathrm{CO}$ transition lines at a spatial resolution of $\sim 4$ pc. With a similar approach using Gaussian curves to fit the [C II] lines they found that the fraction of the [C II] that cannot be attributed to the gas traced by $\mathrm{CO}$ is $20 \%$ around the regions with maxium $\mathrm{CO}$ emission and up to $50 \%$ in the area between the $\mathrm{CO}$ cores. Additionally, they estimate the contribution from ionized gas to be $\leq 15 \%$ in the whole observed region. While these results were obtained with a [C II] map, we only studied regions with strong $\mathrm{CO}$ emission and find that the fraction of [C II] emission associated with $\mathrm{CO}$ differs greatly from region to region. Averaged over all regions we can associate $\sim 54 \%$ with the $\mathrm{CO}$ emission in NGC 4214.

Braine et al. (2012) studied M 33 in the [C II] line with Herschel-HIFI at a spatial resolution of about $50 \mathrm{pc}$ and also found that the $[\mathrm{C} \mathrm{II}]$ lines are broader than the $\mathrm{CO}$ lines by about $50 \%$ but narrower than H I. Spatially resolved [C II] PACS maps (Mookerjea et al. 2011) showed that there is little spatial correlation between [C II] and $\mathrm{CO}$ or $\mathrm{H}$ I.

The difference in [C II] and $\mathrm{CO}$ line profiles is not as striking as in M 33 or the star-forming regions in the SMC for the five regions of NGC 4214 presented in this paper. In regions $\mathrm{I}-1$ and $\mathrm{I}-2$, we find that the $\mathrm{CO}$ is broader by $\sim 20 \%$. Instead, in regions I-3, II, and III, the [C II] line is broader by $\sim 60 \%$. Although comparing the spectra does not directly show 
massive differences in the $\mathrm{CO}$ and [C II] spectra, our decomposition showed that the [C II] emission is associated not only with $\mathrm{CO}$, but also with H I. Studying dwarf galaxies in different tracers with Herschel, Cigan et al. (2016) also found that H I emission peaks correlate with [C II] peaks. We found that in region I the dominance (lowest value of $(72 \pm 13) \%$ found in region I-1) of molecular mass is traced by [C II] rather than $\mathrm{CO}$, implying extended $\mathrm{CO}$-dark molecular gas. However, we note that the quoted values strongly depend on the assumed ISM conditions. Furthermore, we do not resolve the gas clouds spatially as the SOFIA/GREAT beam of $14^{\prime \prime}$ corresponds to a spatial scale of about 200 pc.

Complementary to studies of low-metallicity environment, the origin of [C II] emission was also studied in the Milky Way. The Galactic Observations of Terahertz $\mathrm{C}^{+}\left(\mathrm{GOTC}^{+}\right.$; Yorke et al. 2010) comprises Herschel-HIFI [C II] observations of long lines of sight through the Galactic plane. It was found that the [C II] emission correlates with the star formation rate on galactic scales and that its emission can be divided into contributions from different ISM phases. They attribute $30 \%$ of the luminosity to dense PDRs, $25 \%$ to cold $\mathrm{HI}, 25 \%$ to $\mathrm{CO}$-dark $\mathrm{H}_{2}$, and $20 \%$ to ionized gas (Pineda et al. 2014). Velusamy \& Langer (2014) combined GOTC $+[\mathrm{C} \mathrm{II}]$ data with ancillary $\mathrm{H}$ I and CO data to study the origin of Galactic [C II] emission. They found a widespread [C II] emission. About half of it is associated with diffuse molecular clouds faint in $\mathrm{CO}$, but several lines of sight show features in either $\mathrm{CO}$ or $\mathrm{H}$ I without corresponding emission in [C II].

The star-forming region M $17 \mathrm{SW}$ was studied by Pérez-Beaupuits et al. (2015) using spectrally resolved SOFIA/GREAT [C II] observations and by comparing them to [CI] and low- $J$ CO lines. They analyzed their [C II] velocity channel maps with a different decomposition method and conclude that $\sim 65 \%$ of the mass found with [C II] is not associated with star-forming material. The decomposition of the spectra implies that about $36 \%, 17 \%$, and $47 \%$ of the [C II] emission originates from $\mathrm{HII}, \mathrm{HI}$, and $\mathrm{H}_{2}$ regimes, respectively. Our study does not provide a comparison with a tracer of ionized gas, but previous studies showed that its contribution to the [C II] emission is low. Although there is large scatter, we find that we can associate about $46 \%$ of the [C II] emission on average with the H I emission.

\section{Summary}

We presented [C II] data observed with the GREAT instrument on board the SOFIA telescope in five regions of the lowmetallicity irregular dwarf galaxy NGC 4214. The observations cover three separate regions in NGC 4214 in different evolutionary states. Star formation takes place in regions I and II whereas region III is considered less evolved with no star formation visible. We analyzed the velocity-resolved spectra by decomposing the $[\mathrm{C} \mathrm{II}]$ emission spectra using ancillary $\mathrm{HI}$ and $\mathrm{CO}(2 \rightarrow$ 1) data. We estimated gas masses associated with different ISM phases. We summarize our results as follows:

- The Hi line profiles show almost the same widths of about $30 \mathrm{~km} \mathrm{~s}^{-1}$ in each of the five pointings and thus are broader than the [C II] lines by about $50 \%$ and broader than the $\mathrm{CO}$ lines by $20 \%$ in regions I- 1 and I-2. In regions I-3 and II, the $\mathrm{HI}$ lines are more than twice as broad. All spectra peak at $\sim 300 \mathrm{~km} \mathrm{~s}^{-1}$, except for region III where the peaks are at $\sim 280 \mathrm{~km} \mathrm{~s}^{-1}$. The H I peak positions are shifted to slightly lower velocities in three regions compared to the $[\mathrm{C} \mathrm{II}]$. In addition, the $[\mathrm{C} \mathrm{II}]$ spectrum in this region is very noisy and thus difficult to decompose.

- Using Gaussian curves to fit the [C II], CO, and H I spectra we associate the majority $(>70 \%)$ of the emission with the $\mathrm{CO}$ emission in regions I-1 and I-2. In region I-3, about $65 \%$ of the emission is attributed to the $\mathrm{CO}$ and in region II the fractions are split equally. On average, we can assign 54\% of the [C II] emission to the $\mathrm{CO}$ emission.

- The gas mass of atomic hydrogen is about $10^{6} M_{\odot}$ in each of the five pointings, the molecular gas mass traced by $\mathrm{CO}$ is $\sim 2 \times 10^{5} M_{\odot}$. This is only about $25 \%$ of the molecular mass seen in [C II]. The majority of molecular mass originates from CO-dark gas and between 32 and $87 \%$ of the total [C II] intensity originates from this material. These results agree with most other studies in nearby dwarf galaxies. We find the highest fraction of CO-dark molecular gas mass of $(89 \pm 10) \%$ in region I-2 and it coincides with the position of a super star cluster. In region I, the most evolved starforming region, we find an average $\mathrm{CO}$-dark gas fraction of $84 \%$ while we find less CO-dark molecular gas (63\%) in region II. This region is younger, more metal-rich and more compact. The fraction of CO-dark molecular gas is therefore sensitive to the evolutionary stage of the regions.

- Only about $9 \%$ of the total [C II] emission can be attributed to the CNM in agreement with the findings from Local Group galaxies. There are indications that the $\mathrm{HI}$-associated [C II] also emits from denser phases. Similar to studies of the SMC and $\mathrm{LMC}$, we find prominent wings in the [C II] profiles that cannot be fitted with $\mathrm{CO}$ spectra. The origin of these wings remains poorly constrained.

Acknowledgements. We would like to thank F. Walter for providing us with the OVRO CO(1-0) data, and also Bill Reach for his help in finishing this paper. We also thank the referee for constructive comments that helped improve the quality of the manuscript. K.F., D.C., and F.B. acknowledge support from DFG grant BI 1546/1-1. This study is based on observations made with the NASA/DLR Stratospheric Observatory for Infrared Astronomy (SOFIA). SOFIA is jointly operated by the Universities Space Research Association, Inc. (USRA), under NASA contract NAS2-97001, and the Deutsches SOFIA Institut (DSI) under DLR contract 50 OK 0901 to the University of Stuttgart.

\section{References}

Bolatto, A. D., Wolfire, M., \& Leroy, A. K. 2013, ARA\&A, 51, 207 Braine, J., Combes, F., Casoli, F., et al. 1993, A\&AS, 97, 887 Braine, J., Gratier, P., Kramer, C., et al. 2012, A\&A, 544, A55 Cigan, P., Young, L., Cormier, D., et al. 2016, AJ, 151, 14 Cormier, D., Madden, S. C., Hony, S., et al. 2010, A\&A, 518, L57 Cormier, D., Madden, S. C., Lebouteiller, V., et al. 2014, A\&A, 564, A121 Cormier, D., Madden, S. C., Lebouteiller, V., et al. 2015, A\&A, 578, A53 Dalcanton, J. J., Williams, B. F., Seth, A. C., et al. 2009, ApJS, 183, 67 Dimaratos, A., Cormier, D., Bigiel, F., \& Madden, S. C. 2015, A\&A, 580, A135 Gallagher, III, J. S., \& Hunter, D. A. 1984, ARA\&A, 22, 37

Genzel, R., \& Cesarsky, C. J. 2000, ARA\&A, 38, 761

Glover, S. C. O., \& Mac Low, M.-M. 2011, in EAS Pub. Ser. 52, eds. M. Röllig, R. Simon, V. Ossenkopf, \& J. Stutzki, 147

Goldsmith, P. F., Langer, W. D., Pineda, J. L., \& Velusamy, T. 2012, ApJS, 203, 13

Grenier, I. A., Casandjian, J.-M., \& Terrier, R. 2005, Science, 307, 1292

Guan, X., Stutzki, J., Graf, U. U., et al. 2012, A\&A, 542, L4

Heiles, C., \& Troland, T. H. 2003, ApJ, 586, 1067

Heyminck, S., Graf, U. U., Güsten, R., et al. 2012, A\&A, 542, L1

Hollenbach, D. J., \& Tielens, A. G. G. M. 1999, Rev. Mod. Phys., 71, 173

Hunter, D. A., Gallagher, III, J. S., Rice, W. L., \& Gillett, F. C. 1989, ApJ, 336, 152

Israel, F. P. 1997, A\&A, 328, 471

Kobulnicky, H. A., \& Skillman, E. D. 1996, ApJ, 471, 211

Kulkarni, S. R., \& Heiles, C. 1987, in Interstellar Processes, eds. D. J. Hollenbach, \& H. A. Thronson, Jr., Astrophys. Space Sci. Libr., 134, 87 
K. Fahrion et al.: Disentangling the ISM phases of the dwarf galaxy NGC 4214

Langer, W. D., Velusamy, T., Pineda, J. L., Willacy, K., \& Goldsmith, P. F. 2014, A\&A, 561, A122

Leroy, A. K., Walter, F., Bigiel, F., et al. 2009, AJ, 137, 4670

MacKenty, J. W., Maíz-Apellániz, J., Pickens, C. E., Norman, C. A., \& Walborn, N. R. 2000, AJ, 120, 3007

Madden, S. C., Poglitsch, A., Geis, N., Stacey, G. J., \& Townes, C. H. 1997, ApJ, 483, 200

Maíz-Apellániz, J. 2001, ApJ, 563, 151

Maíz-Apellániz, J., Mas-Hesse, J. M., Munoz-Tunon, C., Vilchez, J. M., \& Castaneda, H. O. 1998, A\&A, 329, 409

Maíz-Apellániz, J., Muñoz-Tuñón, C., Tenorio-Tagle, G., \& Mas-Hesse, J. M. 1999, A\&A, 343, 64

Maíz-Apellániz, J., Cieza, L., \& MacKenty, J. W. 2002, AJ, 123, 1307

McIntyre, V. J. 1997, PASA, 14, 122

Mookerjea, B., Kramer, C., Buchbender, C., et al. 2011, A\&A, 532, A152

Narayanan, D., Krumholz, M., Ostriker, E. C., \& Hernquist, L. 2011, MNRAS, 418,664

Okada, Y., Requena-Torres, M. A., Güsten, R., et al. 2015, A\&A, 580, A54

Pérez-Beaupuits, J. P., Stutzki, J., Ossenkopf, V., et al. 2015, A\&A, 575, A9

Pilbratt, G. L., Riedinger, J. R., Passvogel, T., et al. 2010, A\&A, 518, L1
Pineda, J. L., Langer, W. D., \& Goldsmith, P. F. 2014, A\&A, 570, A121 Planck Collaboration XIX. 2011, A\&A, 536, A19

Poglitsch, A., Krabbe, A., Madden, S. C., et al. 1995, ApJ, 454, 293

Poglitsch, A., Waelkens, C., Geis, N., et al. 2010, A\&A, 518, L2

Requena-Torres, M. A., Israel, F. P., Okada, Y., et al. 2016, A\&A, 589, A28

Schruba, A., Leroy, A. K., Walter, F., et al. 2012, AJ, 143, 138

Sellwood, J. A., \& Balbus, S. A. 1999, ApJ, 511, 660

Stacey, G. J., Geis, N., Genzel, R., et al. 1991, ApJ, 373, 423

Tamburro, D., Rix, H.-W., Leroy, A. K., et al. 2009, AJ, 137, 4424

Thurow, J. C., \& Wilcots, E. M. 2005, AJ, 129, 745

Úbeda, L., Maíz-Apellániz, J., \& MacKenty, J. W. 2007a, AJ, 133, 917

Úbeda, L., Maíz-Apellániz, J., \& MacKenty, J. W. 2007b, AJ, 133, 932

Velusamy, T., \& Langer, W. D. 2014, A\&A, 572, A45

Walter, F., Taylor, C. L., Hüttemeister, S., Scoville, N., \& McIntyre, V. 2001, AJ, 121,727

Walter, F., Brinks, E., de Blok, W. J. G., et al. 2008, AJ, 136, 2563

Wolfire, M. G., Hollenbach, D., \& McKee, C. F. 2010, ApJ, 716, 1191

Yorke, H., Langer, W., Velusamy, T., et al. 2010, in COSPAR Meeting, 38th COSPAR Scientific Assembly, 38, 2482

Young, E. T., Becklin, E. E., Marcum, P. M., et al. 2012, ApJ, 749, L17 


\section{Appendix A: Decomposition: Channel method}

\section{A.1. Description of the method}

In conjunction with the decomposition method described in Sect. 3 that uses Gaussians to fit the spectra, we also used the CO and $\mathrm{H}$ I spectra directly. For each region, we want to describe our [C II] spectra $S$ by a linear combination of $\mathrm{CO}$ and $\mathrm{H} \mathrm{I}$ :

$S_{\mathrm{syn},[\mathrm{CI}]}=a S_{\mathrm{HI}}+b S_{\mathrm{CO}}$.

This method shows some similarities with the approach that was used by Pérez-Beaupuits et al. (2015) to study the origin of [C II] in M17 SW.

To compute the synthetic spectra, we first normalize the $\mathrm{CO}$ and H I spectra by their maximum and then choose the coefficients as percentages so that the $\mathrm{CO}$ and $\mathrm{HI}$ spectra add up to $100 \%$. A percentage $X$ means that the whole H I spectrum is multiplied by this number and then added to the $\mathrm{CO}$ spectrum multiplied correspondingly with $100-X \%$. In an ideal case, this combined synthetic spectrum would peak at the same flux density as the [C II] spectra. However, the best decomposition result might be achieved, if the synthetic spectra peaks at a slightly different flux density. Therefore, we introduce the scaling parameter $k$, where $k=100 \%$ corresponds to a scaling to the height of the $[\mathrm{C} \mathrm{II}]$ peak. Equation (A.1) now reads

$S_{\mathrm{syn},[\mathrm{CI}]}=k \max \left(S_{[\mathrm{C} \mathrm{II}]}\right)\left(X \frac{S_{\mathrm{HI}}}{\max \left(S_{\mathrm{HI}}\right)}+(100-X) \frac{S_{\mathrm{CO}}}{\max \left(S_{\mathrm{CO}}\right)}\right)$.

To quantify the quality of a decomposition with H I percentage $X$ and scaling factor $k$, we compute the residual by subtracting the synthetic from the original [C II] spectrum. The standard deviation of the residual is then used to measure the quality of this decomposition.

This procedure is repeated for all possible values of $X$ with integer percentages and scale factors ranging from $75 \%$ to $100 \%$ for each percentage. For our best decomposition we want a low standard deviation of the residual and a high scaling factor $k$. To achieve the latter, we do not consider values of $k$ below $75 \%$ since we want to reproduce the peak. We then vary the percentages $X$ and scaling factors $k$ and take the $(X, k)$ pair with minimum residual (see Fig. A.1). In this example for region II, $X=40 \%$ and $k=100$.

However, this approach can result in residuals with broad negative features that would correspond to emission not seen in the [C II] spectra. These features are created mostly for high H I percentages as the $\mathrm{HI}$ spectra are broader than the [C II] spectra.

We filter out decompositions with these negative features by rejecting all parameter pairs $(X, k)$ that result in residuals with more than three consecutive velocity channels below a certain threshold. For each pointing, this threshold is determined by the highest noise level of the [C II], $\mathrm{CO}(2 \rightarrow 1)$, and $\mathrm{H}$ I spectra after scaling. While the RMS of the combined spectra depends on the $\mathrm{CO}$ and $\mathrm{HI}$ spectra, the noise in the [C II] also sets a limitation for finding the best decomposition. We find that the best results are obtained when the threshold is set to $1 \sigma \mathrm{rms}$ of the noise.

Using this approach, we can find parameter sets of $X$ and $k$ that give a decomposition for our five [C II] spectra. However, sometimes it is necessary to adjust the parameters manually,
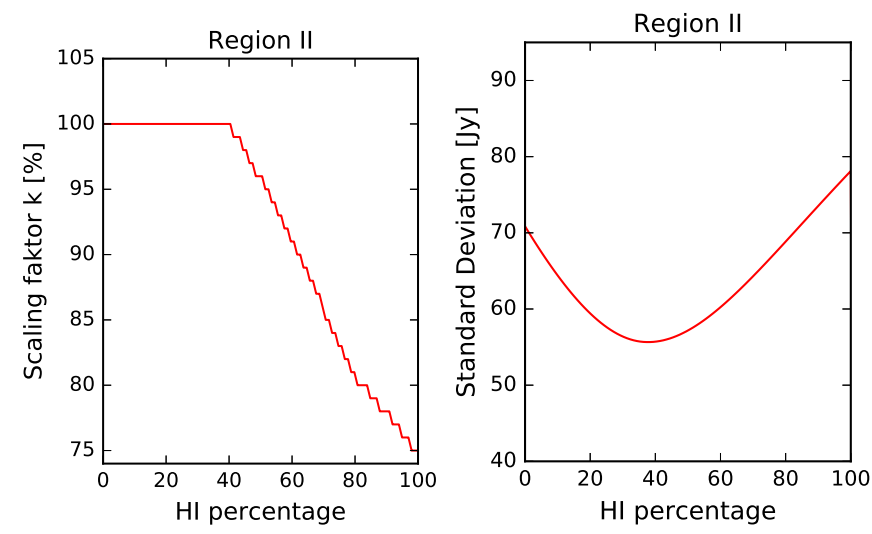

Fig. A.1. First panel: scale factor $k$ that provides the lowest standard deviation of the residual versus the $\mathrm{H}$ I percentage $X$ in region II. Second panel: standard deviation versus $\mathrm{H}$ I percentage. The minimum obtained for an $\mathrm{H}$ I percentage of $37 \%$ and a scaling factor of $100 \%$.

typically within $10 \%$, in order to get the best-matching synthetic spectrum. This range reflects the uncertainty of our channel decomposition method.

\section{A.2. Results}

Table A. 1 reports the best results of the channel method decomposition. We note that the uncertainty of the H I percentages are around $10 \%$. The $\mathrm{CO}(2 \rightarrow 1)$ are $100 \%$ minus the Hi percentages. We also note that the $\mathrm{CO}$ percentage factor is higher in all pointings. The [C II] line profile is more closely associated with the $\mathrm{CO}(2 \rightarrow 1)$ emission than with the broader H I spectra, as was also seen in the Gaussian fit method. Table A.1 also lists the $[\mathrm{C} \mathrm{II}]$ intensity fractions associated with $\mathrm{CO}$ and $\mathrm{HI}$. These values were calculated by direct integration of the $[\mathrm{C} \mathrm{II}]$ and the scaled $\mathrm{CO}$ and $\mathrm{H}$ I spectra.

Figure A.2 presents the final decomposition of our [C II] spectra with the corresponding residuals. The decomposition works best for region I-1 and region II where the weighted spectra of $\mathrm{HI}$ and CO reproduce the shape of the [C II] well. Region III shows a flat residual, but here the best fitting decomposition does not match well owing to the broad, uneven line shape. In this region, it is questionable whether the [C II] emission is strong enough to be decomposed in a useful manner. The decomposition of [C II] in region $\mathrm{I}-3$ looks reasonable, although the residual shows a significant dip at $\sim 290 \mathrm{~km} \mathrm{~s}^{-1}$. We note that it was not possible to reduce this dip any further. The decomposition is worst in region I-2. The weighted spectrum shows side features and has a broad top with two peaks.

Overall, the weighted and scaled spectra of $\mathrm{HI}$ and $\mathrm{CO}(2 \rightarrow 1)$ can account for most [C II] emission at least reasonably well. We find no strong indication for components in the observed regions that are not visible in either $\mathrm{HI}$ or $\mathrm{CO}$, but are visible in [C II].

When comparing the results to those of the Gaussian method (see Table 3), we find no significant differences. However, we prefer the Gaussian fitting method because it enables us to fit several components in the H I spectra. In addition, the channel method requires manual tuning to find the best parameter set for the synthetic [C II] spectrum. 
K. Fahrion et al.: Disentangling the ISM phases of the dwarf galaxy NGC 4214

Table A.1. Results from the channel method decomposition.

\begin{tabular}{cccccc}
\hline \hline Region & $\mathrm{HI}$ & $\mathrm{CO}(2 \rightarrow 1)$ & $k$ & $I_{[\mathrm{C} \mathrm{II}]}(\mathrm{H} \mathrm{I}) / I_{[\mathrm{C} \mathrm{II}]}$ & $I_{[\mathrm{C} \mathrm{II}]}(\mathrm{CO}) / I_{[\mathrm{C} \mathrm{II}]}$ \\
\hline I-1 & $40 \%$ & $60 \%$ & $80 \%$ & $55.0 \%$ & $44.1 \%$ \\
I-2 & $35 \%$ & $65 \%$ & $75 \%$ & $47.8 \%$ & $61.9 \%$ \\
I-3 & $45 \%$ & $55 \%$ & $98 \%$ & $70.6 \%$ & $28.7 \%$ \\
II & $25 \%$ & $75 \%$ & $100 \%$ & $40.4 \%$ & $61.9 \%$ \\
III & $30 \%$ & $70 \%$ & $75 \%$ & $37.5 \%$ & $39.1 \%$ \\
\hline
\end{tabular}

Notes. Intensity fractions $I_{[\mathrm{C} \mathrm{II}]}(\mathrm{HI}) / I_{[\mathrm{C} \mathrm{II}]}$ and $I_{[\mathrm{CH}]}(\mathrm{CO}) / I_{[\mathrm{C} \mathrm{II}]}$ using the scaled $\mathrm{HI}$ and $\mathrm{CO}$ spectra.
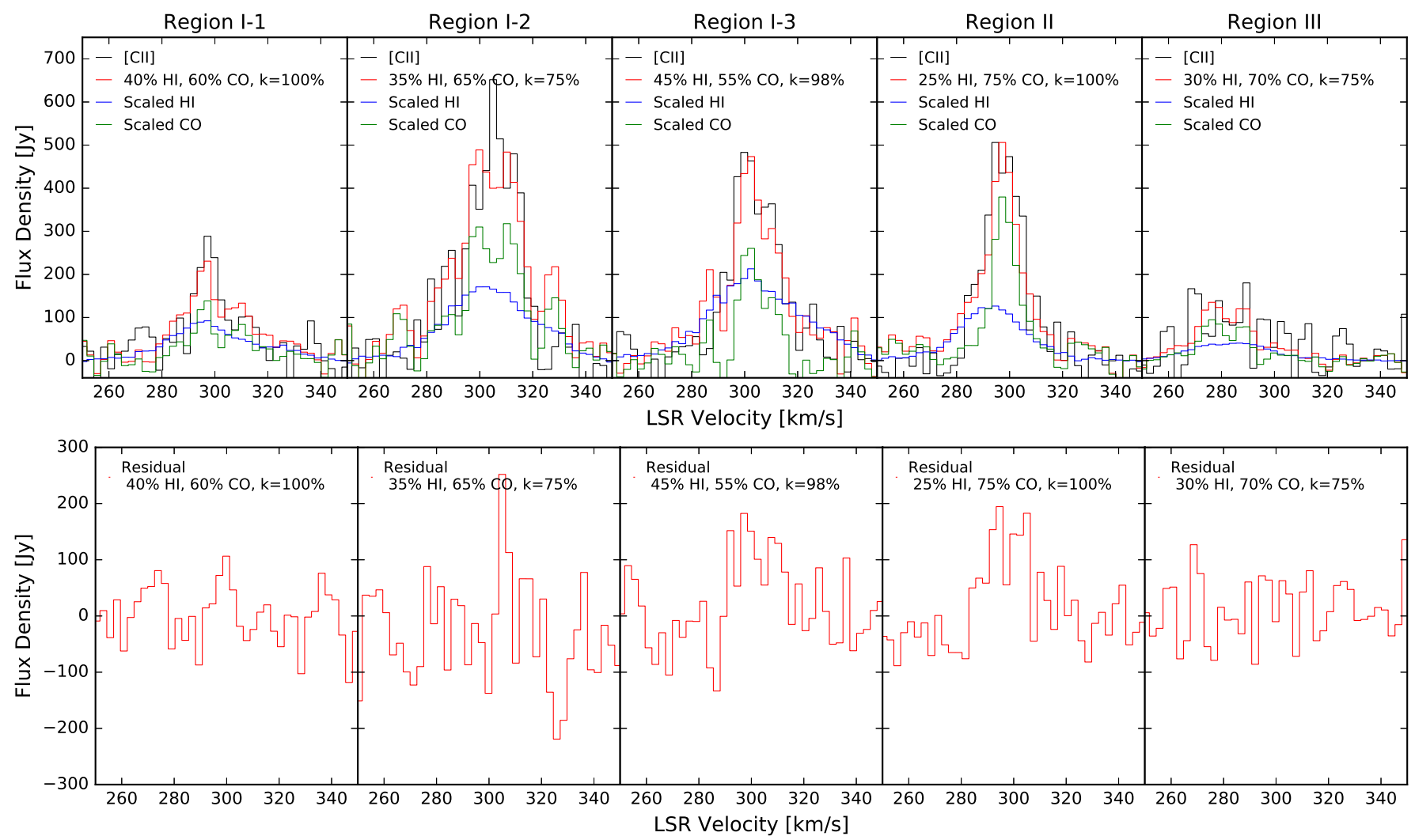

Fig. A.2. Channel method decomposition. Upper row: original [C II] spectra in black and the best fitting synthetic spectra in red with the weightings given in Table A.1. The accordingly scaled $\mathrm{H}$ I spectra are shown in blue, $\mathrm{CO}(2 \rightarrow 1)$ in green. Bottom row: residuals computed from the weighted spectra by subtraction from [C II] data. 